\title{
Management and Outcomes of Bilateral Acetabular Fractures: A Critical Review of the Literature
}

\author{
Vasileios K. Mousafeiris ${ }^{1,2} \cdot$ Anastasia Vasilopoulou $^{1,3} \cdot$ George D. Chloros $^{1,4} \cdot$ Michalis Panteli $^{4}$. \\ Peter V. Giannoudis ${ }^{4,5}$
}

Received: 17 August 2021 / Accepted: 13 December 2021 / Published online: 8 January 2022

(c) The Author(s) 2022

\begin{abstract}
Background Bilateral acetabular fractures constitute a rare entity, and their optimal management is unknown.

Materials and Methods A systematic literature search was conducted in PubMed, Embase and Cochrane Library between 1995 and 2020. Inclusion criteria were studies presenting cases of bilateral acetabular fractures and reporting outcomes. Extracted data included patient demographics, injury mechanism, fracture classification, associated injuries, management and outcomes.

Results Thirty-seven studies (47 cases; 35 males vs 12 females) were included. Mean age was 46 years old (range 13-84) and mean follow-up was 19.8 months (range 1.5-56). High-energy injuries (49\%) and seizures (45\%) were the most common injury mechanisms. Fracture type distribution differed according to injury mechanism. Treatment was surgical in $70 \%$ of cases ( $75 \%$ open reduction and internal fixation vs $25 \%$ acute total hip arthroplasty). Outcomes were excellent/good in $58 \%$ of patients. Complications included heterotopic ossification (11\%), nerve injury (11\%), degenerative arthritis $(6 \%)$, DVT (6\%), and infection (3\%).

Conclusions Bilateral acetabular fractures most commonly occur either after trauma or seizures and are commonly managed operatively. They are not devoid of complications, however, more than half (58\%) achieve complete functional recovery.
\end{abstract}

Keywords Bilateral acetabular fractures $\cdot$ Trauma $\cdot$ Seizures

Peter V. Giannoudis

pgiannoudi@aol.com

Vasileios K. Mousafeiris

v.mousafeiris@yahoo.com

Anastasia Vasilopoulou

vasilopoulou.anastasia85@gmail.com

George D. Chloros

gchlorosdoc@gmail.com

Michalis Panteli

michalispanteli@gmail.com

1 Orthopedic Surgery Working Group, Society for Junior Doctors, Athens, Greece

2 St. Andrews General Hospital of Patras, Patras, Greece

3 Korgialeneio Mpenakeio Hellenic Red Cross Hospital, Athens, Greece

4 Academic Department of Trauma and Orthopaedics, School of Medicine, University of Leeds, Leeds General Infirmary, Clarendon Wing, Floor D, Great George Street, Leeds LS1 3EX, UK

5 NIHR Leeds Biomedical Research Center, Chapel Allerton Hospital, Leeds, UK

\section{Introduction}

Acetabular fractures represent complex injuries, with an incidence of just 3 per 100,000 population/year [1]. Most common causes include road traffic accidents (RTA), followed by falls from height and pedestrians accidents [1]. Because of the high-energy mechanism of injury (MOI), associated injuries are common and are, therefore, related with increased morbidity and mortality [1,2].

Bilateral acetabular fractures on the other hand have an extremely rare occurrence with limited reports in the literature. Little is known about their demographics, MOI, fracture configuration, treatment approach, outcomes, and complications. To our knowledge, there have been no comprehensive reviews of this entity.

The purpose of this study is to summarize the available evidence on bilateral acetabular fractures, including management and outcomes. 


\section{Patients and Methods}

A systematic search of the literature was conducted to identify the available evidence about bilateral acetabular fractures. All studies identified in the English and German published from Jan 1995 to Dec 2020 via the Cochrane Library, Embase via OVID and MEDLINE (through PubMed) electronic databases were assessed. Specific search strings were formulated for each database using the following keywords and/or MeSH terms: (1) ("bilateral"[All Fields] OR "bilaterally"[All Fields] OR "bilaterals"[All Fields]) AND (2) "acetabul*"[All Fields] AND "fractur*"[All Fields] or Bilateral acetabul* fractur*. This study was conducted according to the 2009 Preferred Reporting Items for Systematic Review and Meta-analysis (PRISMA) statement [3].

Inclusion criteria were studies reporting on cases of bilateral acetabular fracture management, outcomes and complications. Exclusion criteria were unilateral acetabular fractures, biomechanical studies, animal studies, review articles, post-mortem studies, foreign language literature, editorials, comments, opinions, letters to the Editor, published abstracts and errata (unless they provide original data). The reference lists of the eligible studies and relevant review articles were cross-checked to identify additional relevant studies.

\section{Data Extraction}

Data extracted from the eligible studies included patient demographics (age, sex, comorbidities), MOI, fracture classification, associated injuries (orthopaedic and non-orthopaedic), procedures performed, classification, outcomes and complications. All data was inserted in an electronic database for subsequent analysis.

\section{Results}

Of the 254 studies screened by title/abstract, 40 papers [4-43] were identified and full text was checked for eligibility. Three case reports $[4,42,43]$ were further excluded at this stage, as they did not report relevant outcomes. Thirtysix case reports [5-33, 35-41] and 1 short case series [34] were finally included and formed the basis of this review (Table 1).

\section{Patient Demographics and MOI (Table 2)}

Forty-seven patients (35 males, 12 females) with a mean age of 46 years (range 13-84 years) were analysed. Followup was reported in $72 \%(34 / 47)$ of the cases with a range of 1.5-56 months, (mean 19.8 months) [5-16, 18-28, 30,
32-41]. The most common MOI was high energy in $49 \%$ $(23 / 47$; male/female ratio $(\mathrm{M} / \mathrm{F})=3.6 / 1)[8,12,13,15,20$, $25,26,29,30,33,34,36,38,39]$, followed by seizures in $45 \%(21 / 47 ; \mathrm{M} / \mathrm{F}=3.2 / 1)[6,9-11,14,16,17,19,21-24$, $27,31,32,35,37,40,41]$, and lastly by insufficiency fractures secondary to osteoporosis $6 \%(3 / 47)$ (Table 2) $[5,7$, 28]. High-energy injuries were secondary to RTAs (70\%; 16 patients) $[12,13,15,20,26,29,30,33,34]$, falls from height (13\%; 3 patients) $[8,38,39]$, direct blows $(9 \% ; 2$ patients) $[25,30]$, crush injury (4\%; 1 patient) [34], and unknown mechanism (4\%; 1 patient) [18]. Mean age was 38 years (range 15-82) for the high-energy group [8, 12, 13, 15, 18, $20,25,26,29,30,33,34,36,38,39], 55$ years (range 13-84) for the seizure group $[6,9-11,14,16,17,19,21-24,27,31$, $32,35,37,40,41]$ and 47 years (range 23-70) for the insufficiency fractures group $[5,7,28]$.

\section{Fracture Classification (Table 3)}

The Letournel and Judet classification [44] was reported in $77 \%$ (36/47) of cases $[6,7,10,12-15,17,18,20,21,25-27$, 29-41], whereas the remaining fractures were not classified $[5,8,11,16,19,22,24-28]$. Overall, the most frequent type was associated both column fracture (ABC) with 18\%, followed by anterior column (AC) with $17 \%$, while the least common was posterior wall and column (PWC) with $4 \%$ and T-shaped (T) with $3 \%$.

For the high-energy injuries, the most common fracture types on either side were PW [12, 18, 20, 34, 38] and TPW $[13,20,34,38]$ with $24 \%$ each, whereas, the most common fracture combination was bilateral TPW (18\%) [20, 34, 38], followed by AW on one side and PW on the contralateral side $(14 \%)$ [13, 34]. In the seizure group, the most common fracture types on either side were $\mathrm{ABC}(40 \%)[6,21,31$, $32,37,40,41]$, followed by AC $(23 \%)[10,14,27,37]$. The most common fracture combination was bilateral $\mathrm{ABC}$ in $31 \%$ of cases $[6,21,40,41]$, whereas other combinations were inconsistent, in the sense that in the remaining cases, the fracture on the right is different from the fracture on the left $[9-11,14,16,17,19,22-24,27,31,32,35,37]$. For the Insufficiency fractures group, only 1 fracture combination is reported: AC and posterior hemitransverse [7].

\section{Associated Injuries}

Those were reported in $89 \%$ of the cases (42/47 cases) [5-14, 16, 17, 19-24, 26-34, 36-41]. The most common associated injuries were upper limb injuries $(26 \%)[8,10$, $13,17,19,31,32,34,37,39]$, pelvic fractures $(23 \%)$ [7, $11,12,34,36,38,39,41]$, femoral fractures $(19 \%)[10,16$, $31,32,34,38]$, spine injuries $(14 \%)$ [10, 27, 30, 31, 34], acetabular fracture associated with hip dislocation $(23 \%)$ 
Table 1 PRISMA flow diagram

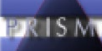

PRISMA 2009 Flow Diagram
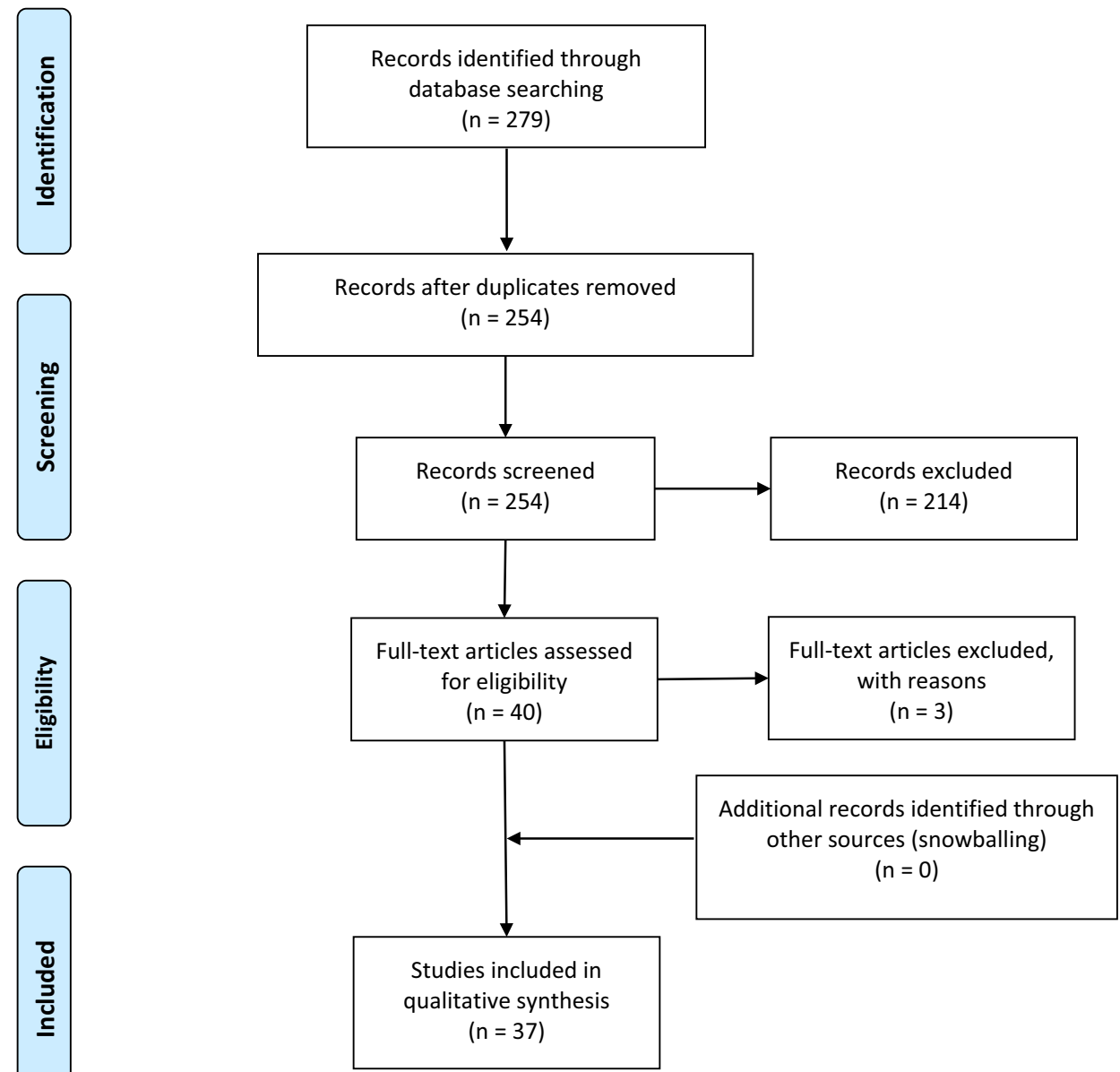

Table 2 Patient demographics and mechanism of injury

\begin{tabular}{llll}
\hline Mechanism of injury & $\%$ (number of cases) & $\begin{array}{l}\text { Male/ } \\
\text { female } \\
\text { ratio }\end{array}$ & $\begin{array}{l}\text { Mean age in } \\
\text { years (range) }\end{array}$ \\
\hline $\begin{array}{l}\text { 1. High-energy injuries } \\
\text { Total }\end{array}$ & $39 \%(23)$ & $3.6 / 1$ & $38(15-82)$ \\
RTA & $70 \%(16)$ & & \\
Falls & $13 \%(3)$ & & \\
Direct blow & $9 \%(2)$ & & \\
Crush injury & $4 \%(1)$ & & \\
Not reported & $4 \%(1)$ & & \\
2. Seizures & $45 \%(21)$ & $3.2 / 1$ & $55(13-84)$ \\
Total & $6 \%(3)$ & $1 / 2$ & $47(23-70)$ \\
3. Insufficiency fractures & \\
\multicolumn{2}{l}{ Total } &
\end{tabular}

$[10,14,20,22,23,26,33,38,40]$, chest injuries (12\%) [13, $29,34,36]$, lower leg fractures $(7 \%)[30,34,36]$, and head injury (2\%) [26].

\section{Treatment}

Operative treatment versus non-operative treatment was undertaken in $70 \%[5-8,10,12-18,20,21,23,27,28,31$, $33,34,36-40]$ and $30 \%[9,11,18,19,22,25,26,29,30,32$, $35,41]$ of cases, respectively.

\section{Operative Management}

Operative management of bilateral acetabular fractures has been reported as a single-stage, a 2-stage or even a more than 2-stage procedure.

Overall, single-stage fixation was reported in $52 \%$ of cases $[7,8,13,16,17,20,23,27,34,36,37,39]$, a 2-stage 


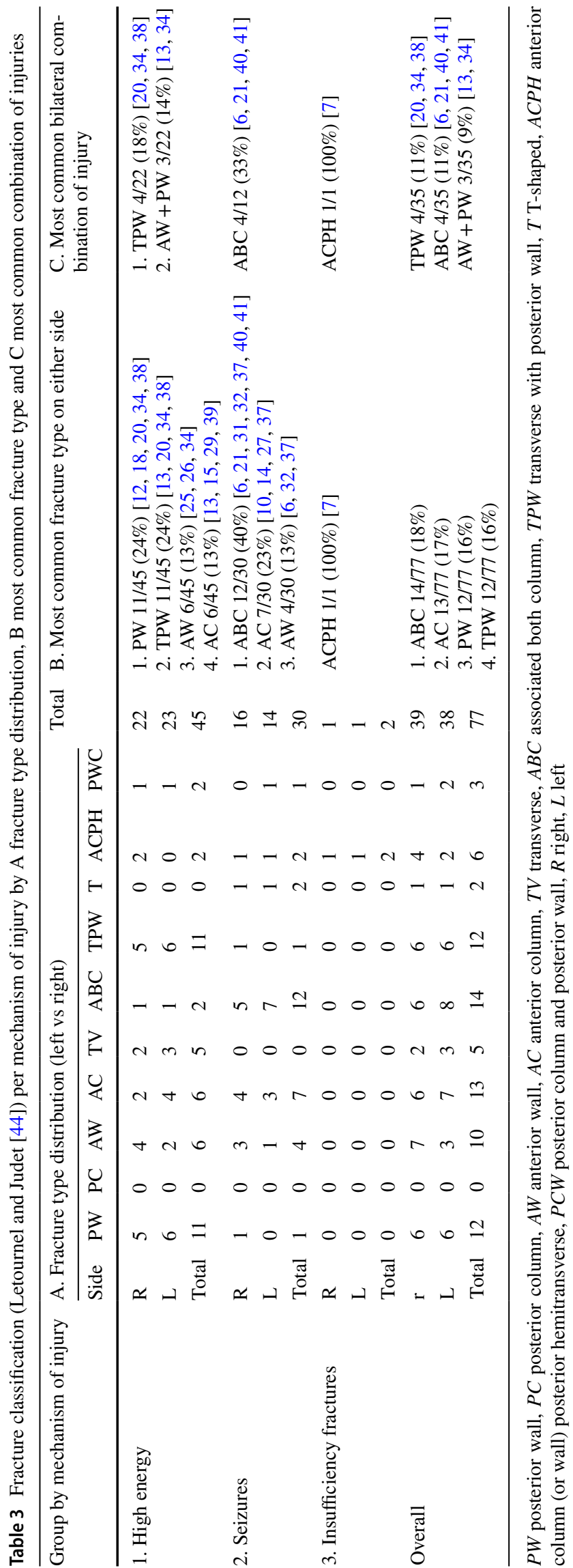

in $45 \%$ of cases $[5,10,12-15,21,26,28-30,33,34,38$, $40]$, whereas in 1 case $(3 \%)$ a 4 -staged operative management [6] was reported (Table 4). The mean time elapsed between stages ranged from 1 day to 3 months $[5,10$, 12-15, 21, 26, 28-30, 33, 34, 38, 40].

In the high-energy group, $17 \%(4 / 23)$ of cases were treated conservatively $[25,26,29,30]$. Of the $83 \%(19 / 23)$ of cases that were treated operatively $[8,12,13,15,18$, $20,33,34,36,38,39], 90 \%$ (18/19) give further details on stages of fixation $[8,12,13,15,20,25,26,29,30,33$, $34,36,38,39]$. Fifty-six percent of cases $(10 / 18)$ were treated in a single stage $[8,13,20,34,36,39]$, while a 2 -stage procedure was undertaken in $8 / 18$ cases $(44 \%)$ [ 12 , $15,33,34,38]$.

In the seizure group, $48 \%(10 / 21)$ of cases were treated conservatively $[9,11,19,22,24,32,35,41]$. Of the $52 \%$ $(11 / 21)$ of cases that were treated operatively $90 \%(10 / 11)$ give further details on stages of fixation $[6,9-11,14,16$, 17, 19, 21-24, 27, 31, 32, 35, 37, 40, 41]. Single-staged procedure was performed in $50 \%(5 / 10)[16,17,23,27$, 37], 2- staged procedure was performed in $40 \%$ (4/10) [10, $14,21,40]$ and 4 -staged procedure was carried out in $10 \%$ (1/10) [6].

In the insufficiency fractures group 33\% (1/3) cases were treated via single-stage procedure [7], while $67 \%(2 / 3)$ cases were treated via 2 -staged procedure $(5,28)$.

\section{Type of Surgery and Surgical Approach Used (Tables 5 and 6)}

The majority of the fractures in the high-energy group (75\%) were treated with open reduction and internal fixation (ORIF) [8, 12, 13, 15, 18, 20, 33, 34, 36, 38, 39], with the remaining $25 \%$ being treated conservatively [25, 26, 29 , 30]. No total hip arthroplasty (THA) was performed in the high-energy group (Table 5) [8, 12, 13, 15, 18, 20, 25, 26, $29,30,33,34,36,38,39]$.

In the seizure group, the majority of fractures was treated conservatively (54\%), with $25 \%$ undergoing ORIF [6, 10, $17,21,23,27,31]$ and $21 \%$ treated with THA $[6,14,16,37$, 40]. In this group, 4 cases were treated initially conservatively and then received late THA bilaterally (Table 5) [6, $14,37,40]$.

In the insufficiency fractures group 50\% of the fractures underwent THA [5, 28], 25\% were treated conservatively, and $25 \%$ underwent ORIF [7]. One case originally managed conservatively, underwent THA at a later stage (Table 5) [5].

Overall, five cases were treated initially conservatively, but then received late THA bilaterally $[5,6,14,37,40]$. There were, also, three cases where the two sides were treated with a different procedure [10,34]. More specifically, in the study of Gill et al. [10] the patient underwent ORIF on the right side and THA on the left side, due to an 
Table 4 Staging in operatively treated bilateral acetabular fractures per MOI group

\begin{tabular}{lccl}
\hline MOI group & Single stage & 2-stages & $>2$ stages \\
\hline High energy & $10(56 \%)$ & $8(44 \%)$ & $0(0 \%)$ \\
Seizures & $5(50 \%)$ & $4(40 \%)$ & $1(10 \%)$ \\
Insufficiency fractures & $1(33 \%)$ & $2(67 \%)$ & $0(0 \%)$ \\
OVERALL & $16(52 \%)$ & $14(45 \%)$ & $1(3 \%)$ \\
\hline
\end{tabular}

intracapsular neck femur fracture. In two cases in the study of Steven et al. [34], the patients underwent ORIF on the right side and were treated conservatively on the left side.

In the high-energy group, $68 \%$ of the fractures underwent a posterior approach $[13,18,33,34,38]$, while $32 \%$ were approached anteriorly $[8,12,15,34]$. In the seizure group $40 \%$ of the fractures underwent anterior approach [23, 27], followed by $40 \%$ that underwent posterior $[6,21]$ and $20 \%$ that underwent combined approach [21]. In the insufficiency group, all fractures underwent anterior approach [7] and the approach was not reported in 3 cases treated with ORIF (Table 6) [10, 17, 31].

\section{Length of Surgery, Need for Blood Transfusion and ICU Stay}

Length of surgery was not reported throughout the studies. The need for blood transfusion is reported in only five patients $[11,13,24,35]$ and a mean of four units of packed red blood cells (range 3-6 units) is reported in two of those patients $[24,35]$. Post-operative ICU requirement was reported in only eight patients [10, 11, 13, 23, 24, 29, 41]; the mean length of stay was 3.2 days [1-7 range], while in three cases $[10,13,41]$ length of ICU stay is not reported.

\section{Rehabilitation}

Rehabilitation protocol was reported in 15 cases [6, 9, $11-13,15,21-23,26,32,33,35,37,39] .9$ cases [6, 9, $11,21-23,32,35,37]$ in the seizure group and 6 cases [12, $13,15,26,33,39]$ in the high-energy group, while in the remaining cases, no post-operative physiotherapy and weightbearing protocols are reported $[5,7,8,10$, $14,16-20,24,25,27-31,34,36,38,40,41]$. In those reported, no patient was allowed to immediate fully weight bear. Patients were advised to avoid weight-bearing for at least 6 weeks in the majority of studies $[12,15,21,23$, $33,35]$.

\section{Outcomes}

Outcomes were reported descriptively, i.e. excellent/good vs fair vs poor in $85 \%(40 / 47)$ of cases [5-18, 20-26, 28, 30-40], whereas in the rest, no outcomes were reported [19, 27, 29, 34, 41].

Overall, there were 58\% excellent/good outcomes with complete functional recovery and independent mobilization [5, 7, 9, 10, 13, 15-18, 22, 25, 26, 28, 31, 33-35, 38-40], $22 \%$ fair with limited range of motion of both hips and moderate pain $[6,8,23,30,32,34,36,37]$, and $20 \%$ poor
Table 5 Treatment modality per MOI group per fracture

\begin{tabular}{|c|c|c|c|c|c|c|c|c|c|}
\hline \multirow{2}{*}{$\begin{array}{l}\text { Side } \\
\text { Treatment }\end{array}$} & \multicolumn{3}{|l|}{ Right } & \multicolumn{3}{|l|}{ Left } & \multicolumn{3}{|l|}{ Combined } \\
\hline & ORIF & THA & Cons & ORIF & THA & Cons & ORIF & THA & Cons \\
\hline High energy & 16 & 0 & 4 & 14 & 0 & 6 & $30(75 \%)$ & $0(0 \%)$ & $10(25 \%)$ \\
\hline Seizures & 7 & 5 & 14 & 6 & 6 & 14 & $13(25 \%)$ & $11(21 \%)$ & $28(54 \%)$ \\
\hline Insufficiency fractures & 1 & 2 & 1 & 1 & 2 & 1 & $2(25 \%)$ & $4(50 \%)$ & $2(25 \%)$ \\
\hline Overall & 24 & 7 & 19 & 21 & 8 & 21 & $45(45 \%)$ & $15(15 \%)$ & $40(40 \%)$ \\
\hline
\end{tabular}

ORIF open reduction and internal fixation, THA total hip arthroplasty, Cons conservative

Table 6 Overall prevalence of surgical approaches used per MOI group and fracture

\begin{tabular}{|c|c|c|c|c|c|c|c|c|}
\hline \multirow[t]{2}{*}{ Groups } & \multicolumn{3}{|c|}{ Anterior approaches } & \multicolumn{3}{|l|}{ Posterior approaches } & \multirow{2}{*}{$\begin{array}{l}\text { Combined } \\
\text { approach }\end{array}$} & \multirow[t]{2}{*}{ Overall } \\
\hline & $\begin{array}{l}\text { Modified } \\
\text { Stoppa/AIP }\end{array}$ & Ilioinguinal & Iliofemoral & Kocher-Langenbeck & Iselin & Moore & & \\
\hline High energy & $5(18 \%)$ & $3(11 \%)$ & $1(4 \%)$ & $16(57 \%)$ & 0 & $3(11 \%)$ & 0 & $28(70 \%)$ \\
\hline Seizures & $2(20 \%)$ & $2(20 \%)$ & 0 & $2(20 \%)$ & $2(20 \%)$ & 0 & $2(20 \%)$ & $10(25 \%)$ \\
\hline Insufficiency fractures & 2 & 0 & 0 & 0 & 0 & 0 & 0 & $2(5 \%)$ \\
\hline Total & $9(23 \%)$ & $5(13 \%)$ & $1(3 \%)$ & $18(45 \%)$ & $2(5 \%)$ & $3(8 \%)$ & $2(5 \%)$ & $40(100 \%)$ \\
\hline
\end{tabular}

Anterior approach: Ilioinguinal, iliofemoral or modified Stoppa approach. Posterior approach: Kocher-Langenbeck, Iselin or Moore. Combined approach refers to both anterior and posterior approaches done during the same stage 
outcomes with limited activity and walking ability (Table 7) [11, 12, 14, 20, 21, 24, 34].

According to injury group, excellent/good outcomes were reported in $60 \%$ of the high-energy patients $[13,15,18,25$, $26,33,34,38,39], 47 \%$ of the seizures patients [9, 10, 16, $17,22,31,35,40]$, and in $100 \%$ of the insufficiency fracture group $[5,7,28]$.

Functional scores were reported in 30\% (14/47) of cases $[13,16,18,21,22,28,34,37]$. The mean Harris hip score reported in $43 \%(6 / 14)$ of cases was 85 (good) [13, 16, 21, $28,37]$ and the Oxford Hip Score reported in $43 \%(6 / 14)$ of cases was 20-29 in 1 case (moderate to severe arthritis), 30-39 in 2 cases (mild to moderate arthritis) and 40-48 in 3 cases (satisfactory joint function) [34]. The Merle D'Aubigne and Postel Criteria were reported in 14\% (2/14) cases as good [18, 22].

We examined whether the presence of associated pelvic injury has any impact on the outcome of patients with bilateral acetabular fractures. Eight papers report on associated pelvic injuries $[7,11,12,34,36,38,39$, 41]. However, no association was noted as pelvic injury did not affect outcome.

\section{Complications}

Complications were only reported in $36 / 47$ cases $(77 \%)$ [5-8, 12-17, 20-26, 28, 30, 32-40]. The most common complication is heterotopic ossification 4/36 (11\%) [6, 21, $33,37]$ and nerve injury $4 / 36$ (11\%) [12, 20, 33, 36], followed by degenerative arthritis $2 / 36(6 \%)$ [6, 30], DVT $2 / 36(6 \%)[14,36]$, infection 1/36 (3\%) [8] and abductor muscle weakness 1/36 (3\%) [24] (Table 8). Finally, mortality was only reported in two cases and was unrelated to surgery [41].

\section{Discussion}

Acetabular fractures represent complex injuries, usually associated with high-energy trauma and remain a challenge to manage $[1,2,45,46]$. The literature is scarce regarding bilateral injuries, with only 47 cases from 37 studies being identified. The results show a male preponderance of $75 \%$, which is similar to the unilateral injuries [45-47]; this is comparable in both high-energy and seizure groups $(78 \%$ and $76 \%$ ). As in the case of unilateral injuries, a big proportion of bilateral injuries are the result of a high-energy mechanism $(49 \%)$ in the younger population with a mean age of 38 years. However, this study also identified that a significant proportion (45\%) of these injuries occurs secondary to seizures, mainly involving an older population with a mean age of 55 years old. Finally, only 3 cases of bilateral insufficiency fractures have been reported (Table 2) [5, 7, 28]. Interestingly, there was a 23 -year-old female patient with pregnancy-induced osteoporosis in the latter group [5]. The reported mean age in the unilateral literature is closer to the high-energy group, a finding which is not surprising as they share a common mechanism [45-48]. The Letournel and Judet classification system [44] remains the most commonly used classification system for acetabular fractures [48, 49], utilised by all included papers that report on classification [6, 7, 10, 12-15, 17, 18, 20, 21, 25-27, 29-41].

The most common fracture types in our study were ABC, AC, TPW and PW with frequencies similar to those

Table 7 Outcomes according to MOI

\begin{tabular}{llll}
\hline Groups & \multicolumn{2}{l}{ Descriptive outcomes-40 cases } & \\
\cline { 2 - 4 } & Excellent/good & Fair & Poor \\
\hline High energy & $12[13,15,18,25,26,33,34,38,39](60 \%)$ & $5[8,30,34,36](25 \%)$ & $3[12,20,34](15 \%)$ \\
Seizures & $8[9,10,16,17,22,31,35,40](47 \%)$ & $4[6,23,32,37](24 \%)$ & $5[11,14,21,24](29 \%)$ \\
Insufficiency fractures & $3[5,7,28](100 \%)$ & 0 & 0 \\
Overall & $23(58 \%)$ & $9(22 \%)$ & $8(20 \%)$ \\
\hline & Functional scores-14 cases & Oxford Hip Score & Merle D'Aubigne and Postel \\
\cline { 2 - 4 } & Harris hip Score & & Criteria \\
\hline High-energy & $2[13]$ & $6[34]$ & $1[18]$ \\
Seizures & $3[16,21,37]$ & 0 & $1[22]$ \\
Insufficiency fractures & $1[28]$ & 0 & 0 \\
Overall & 6 & 6 & 2 \\
\hline
\end{tabular}


reported in the literature $[1,45,46,50-52] . P W C$ and T-shaped were the least common, whilst no cases with PC fractures we reported. In the high-energy group, the most common fracture types were similar to those reported in the literature (PW and TPW 24\% each, 48\% combined) $[45,46]$. In the seizure group, the most common fracture types included the anterior wall and column (AW, $\mathrm{AC}$ and $\mathrm{ABC}$ fractures; $13 \%, 23 \%$ and $40 \%$ respectively, $75 \%$ combined). Although AC fractures are not as frequent in the unilateral population, their frequency has been reported to be higher in the elderly population [53], which may explain their higher incidence in the seizure group. Furthermore, contrary to the literature, AW fracture in our study was reported almost eight times more compared to the acetabular fractures in general $[46,52]$. Even comparing with the elderly population (aged $>60$ ), this still remains about four times more [53]. Lastly, in the insufficiency fractures group, ACPH was the only type reported, in agreement with Papadakos et al. [54], where ACPH was the most common type in acetabular fractures attributed to low energy mechanisms. As far as the different combinations of the bilateral injuries, the high-energy group had TPW and AW + PW, in $18 \%$ and $14 \%$ of cases, respectively. In contrast, the seizure group showed an $\mathrm{ABC}$ pattern in the majority of cases (33\%), see Table 4. Given the simultaneous continuous bilateral contraction of the strong pelvi-trochanteric musculature during a seizure episode $[35,49,55]$, which forces the femoral head medially [56], the authors hypothesized that, in the seizure group, the fractures are possibly added every few seconds of the seizure episode in a cumulative way.

More than $40 \%$ of acetabular fractures have associated injuries, with the most common being lower extremity (21.8\%), followed by upper extremity (9.6\%), and pelvis/ spine in $5.5 \%$, whereas multiple fractures are reported in $22.8 \%$ of cases [46]. In this study, the majority of associated injuries were upper extremity (26\%), followed by pelvic (23\%), femur (19\%) and spinal (14\%) fractures. Of note, while in the high-energy group similar frequencies are shown compared to the general literature, in the seizure group, the incidence of associated upper extremity, femoral

Table 8 Complications reported in 75\% (36/47) of cases

\begin{tabular}{ll}
\hline Complications & $\begin{array}{l}\text { Frequency } \% \\
\text { (nr. of cases) }\end{array}$ \\
\hline Heterotopic ossification $[6,21,33,37]$ & $11 \%(4)$ \\
Nerve injury $[12,20,33,36]$ & $11 \%(4)$ \\
Degenerative arthritis $[6,30]$ & $6 \%(2)$ \\
DVT [14, 36] & $6 \%(2)$ \\
Infection [8] & $3 \%(2)$ \\
Abductor muscle weakness [24] & $3 \%(1)$ \\
\hline
\end{tabular}

fractures and spine injuries was $64 \%, 62 \%$ and $50 \%$, respectively. This is commensurate with a recent meta-analysis which showed an increased general fracture risk in patients with epilepsy compared to the general population, particularly with hip, forearm and spine fractures [57]. Finally, chest injuries showed a similar prevalence, whereas head injuries were underreported in the literature [46]. The authors tried to assess whether the treatment approach changes with the nature and presence of associated injuries. However, no pattern was identified regarding differentiation of the treatment approach based on the presence or absence of pelvic or hip injuries. For example, all four cases with sacral fractures, as well as all three cases that had pelvic fractures were treated differently and in the case of hip fractures, almost half received ORIF and half received THAs.

Treatment options varied and included conservative vs surgical (ORIF or THA with either single- or multi-staged operations for both sides). Overall, 45\% underwent ORIF, with $40 \%$ treated either initially or ultimately conservatively, while the rest (15\%) underwent THA. Interestingly, almost half of the cases in both the high energy and the seizure group were treated with single-stage procedures and the other half with two-staged procedures, revealing no difference in treatment choice regarding the injury mechanism and the different fracture patterns involved (single-staged vs 2 -staged; $56 \%$ vs $44 \%$ in high-energy group, $50 \%$ vs $40 \%$ in seizure group and $52 \%$ vs $45 \%$ overall). ORIF remains the "gold standard" treatment for both unilateral and bilateral acetabular fractures [45, 46, 49]. The Kocher-Langenbeck was the most commonly used approach $(45 \%)$ in our study, in agreement with the literature (48.7-54.8\%) [46, 52]. Moreover, the Stoppa approach was utilized in almost a quarter of our patients (23\%), almost 6 times more frequently than in the unilateral literature $[46,52]$. Although, the Ilioinguinal approach is reported as the second most common approach $(21.9-25.9 \%)$ [46, 52], in our study it was performed much less frequently (13\%). The iliofemoral approach and combined approaches were performed slightly less frequently (Iliofemoral: $3 \%$ vs $1.1-12.4 \%$ and combined: $5 \%$ vs $9.2-10.1)$ [46, 52]. Of note, $70 \%$ of the ORIF procedures were performed in the high-energy group, almost three times more than those reported in the seizure group (25\%).

THA was the treatment of choice in $15 \%$ of the total fractures of our study but was only performed in the seizure group. More specifically, four cases from the seizure group were treated initially conservatively, and then underwent a late THA. This may be explained by the fact that the highenergy trauma cases are higher impact injuries and require more urgent treatment with ORIF, whereas in seizure group the injury is lower impact, so more patients are treated initially conservatively and receive elective THAs afterwards. In line with that, Papadakos et al. reported that $21.1 \%$ of 
the low energy mechanism acetabular fractures were treated initially conservatively and subsequently received late THA [54]. In unilateral acetabular fractures in the elderly, especially when femoral head fractures are present, early stage THA has shown good clinical results when combined with stable fracture fixation $[58,59]$. In addition, THA is a treatment option for secondary arthritis due to non-union, malunion of previously conservatively managed or operated acetabular fractures.

Non-operative treatment is usually reserved among others, for minimally displaced fractures, for patients with significant comorbidities and for the elderly with severe osteoporosis $[48,56,60]$. In this study, almost half of the patients in the seizure group were treated conservatively (48\%), while another $19 \%$ were treated initially conservatively and received late THAs. In contrast, only $17 \%$ of the high-energy group patients were treated conservatively and in another $9 \%$, one side was treated operatively and the other non-operatively. In line with that, Papadakos et al. [54] reported that in low energy mechanism acetabular fractures, $39.4 \%$ were treated conservatively and another $21.1 \%$ were treated initially conservatively and then received THA, while only $31 \%$ underwent ORIF. It is not known why more patients in the seizure group were treated conservatively, but it can be assumed that the risk for ORIF hardware failure or THA dislocation during a subsequent seizure episode and the poor bone quality both as side effect of the antiepileptic medications and due to their increased age, as well as increased medical comorbidities and perioperative morbidity led the surgeons to stall operative treatment. Nevertheless, the choice of treatment approach is frequently directed by the fracture pattern, so a change in the fracture proportion would mandate a change in the approach used.

As far as rehabilitation, this has been reported in 15 cases $[6,9,11-13,15,21-23,26,32,33,35,37,39]$, including 9 cases in the seizure group $[6,9,11,21-23,32,35,37]$ and 6 in the high-energy group $[12,13,15,26,33,39]$. The majority of patients were advised to avoid full weight-bearing for at least 6 weeks [12, 15, 21, 23, 33, 35].

Excellent/good functional outcomes are reported in 58\% of the cases, which is significantly lower than in the unilateral literature (73-80\%) [46, 47, 52, 60, 61]. Although, due to short-term follow-up period ( $<2$ years) further improvement or deterioration of the outcomes cannot be excluded, Giannoudis et al. [52] reported that studies with longer follow-up had better functional outcomes. In this study, fair and poor outcomes are reported in $23 \%$ and $20 \%$ respectively, compared to the unilateral literature (20\% vs $27 \%$ ) [46, 47 , $52,60,61]$.

The most common complications reported included traumatic nerve palsy in $11 \%$ of cases, and heterotopic ossification in another $11 \%$. These occurred less frequently than in unilateral acetabular fractures [16\% [52] and 19-25.6\% [46, 52]], respectively. In addition, post-traumatic osteoarthritis had an incidence of $6 \%$ in this series compared to $17.6-19.8 \%$ reported in the literature $[46,52]$, which may be attributed to the short-term ( $<2$ year) follow-up in the reported studies. Remaining complications including DVT (6\%), local infection (3\%) and abductor muscle weakness (3\%) are reported, which are comparable to the reported literature in unilateral injuries (DVT: 4.3-5.2\%, local infection: $4.4-4.5 \%)[46,52]$.

This study has limitations. The majority of the included studies are case reports and therefore of low quality. Another limitation is that no study reports on ISS (injury severity score), which is an important factor in deciding type and timing of treatment. The exact mode of treatment is also not uniform in the included studies. Interestingly, $16 \%$ of the cases do not report whether the operations were performed in a single or multiple stages. Regarding combined pelvic and acetabular fractures or other associated orthopaedic injuries there are no protocols established defining early and definitive fixation strategy. In addition, length of surgery and intra-operative blood loss is not reported in any study. The need for blood transfusion is reported in only five patients. Rehabilitation protocol is reported in 15 cases with no clear functional protocols.

\section{Conclusion}

Bilateral acetabular fractures are complex injuries which remain a challenge to manage. They are, mostly, caused by high-energy injuries or seizures. Fracture types of the highenergy group are similar to the unilateral types reported in the literature, while those of the seizure group similar to the types reported mostly in the elderly. ORIF remains the standard method of treatment of the high-energy group, similar to the unilateral fractures, while in the seizure group, bilateral fractures are treated either conservatively, with ORIF, or THA. Larger series that address the aforementioned limitations of the literature are needed to provide further insight and guidelines for orthopaedic trauma surgeons managing bilateral acetabular fractures.

\section{Declarations}

Conflict of interest The authors declare that they have no conflict of interest. 
Ethical standard statement This article does not contain any studies with human or animal subjects performed by the any of the authors.

Informed consent For this type of study, informed consent is not required.

Open Access This article is licensed under a Creative Commons Attribution 4.0 International License, which permits use, sharing, adaptation, distribution and reproduction in any medium or format, as long as you give appropriate credit to the original author(s) and the source, provide a link to the Creative Commons licence, and indicate if changes were made. The images or other third party material in this article are included in the article's Creative Commons licence, unless indicated otherwise in a credit line to the material. If material is not included in the article's Creative Commons licence and your intended use is not permitted by statutory regulation or exceeds the permitted use, you will need to obtain permission directly from the copyright holder. To view a copy of this licence, visit http://creativecommons.org/licenses/by/4.0/.

\section{References}

1. Laird, A., \& Keating, J. (2005). Acetabular fractures: A 16-year prospective epidemiological study. The Journal of bone and joint surgery British volume., 87(7), 969-973.

2. Matta, J. M. (1996). Fractures of the acetabulum: Accuracy of reduction and clinical results in patients managed operatively within three weeks after the injury. Journal of Bone and Joint Surgery. American Volume, 78(11), 1632-1645.

3. Moher, D., Shamseer, L., Clarke, M., Ghersi, D., Liberati, A., Petticrew, M., et al. (2015). Preferred reporting items for systematic review and meta-analysis protocols (PRISMA-P) 2015 statement. Systematic Reviews., 4(1), 1.

4. Alenazi, B., Rana, A.-Q., \& Vaid, H. M. (2013). The importance of imaging for status epilepticus patients to rule out fractures-A case report. Journal of Taibah University Medical Sciences., 8(2), 120-122.

5. Aynaci, O., Kerimoglu, S., Ozturk, C., \& Saracoglu, M. (2008). Bilateral non-traumatic acetabular and femoral neck fractures due to pregnancy-associated osteoporosis. Archives of Orthopaedic and Trauma Surgery., 128(3), 313-316.

6. Balcarek, P., Dresing, K., Walde, T. A., Tezval, M., \& Stürmer, K. M. (2009). Myoclonus-induced bilateral acetabular fracture dislocations. Journal of Arthroplasty, 24(5), 826.e11-5.

7. Benz, D., Lim, P., \& Balogh, Z. J. (2019). Acute atraumatic bilateral acetabular insufficiency fractures. Journal of Orthopaedic Surgery (Hong Kong), 27(2), 2309499019858574.

8. Chouhan, V. (2016). Qivas Therapy for treatment of implant associated MDR bacterial infection. Journal of Orthopaedic Case Reports., 6(2), 69-71.

9. Friedberg, R., \& Buras, J. (2005). Bilateral acetabular fractures associated with a seizure: A case report. Annals of Emergency Medicine, 46(3), 260-262.

10. Gill, J. R., Murphy, C. G., Quansah, B., \& Carrothers, A. D. (2015). Seizure induced polytrauma; not just posterior dislocation of the shoulder. BMJ Case Reports., 2015, bcr2015211445.

11. Granhed, H. P., \& Karladani, A. (1997). Bilateral acetabular fracture as a result of epileptic seizure: A report of two cases. Injury, 28(1), 65-68.

12. Hardy, S. L. (1997). Femoral nerve palsy associated with an associated posterior wall transverse acetabular fracture. Journal of Orthopaedic Trauma, 11(1), 40-42.
13. Hassan, B., Abdelhalim, E., Mohammed, E., Mohammed, S., \& Abdelmajid, E. (2015). Bilateral acetabular fracture-two cases report. International Journal of Medicine and Surgery., 2(1), $19-22$.

14. Heyer, J. H., Thakkar, S. C., Zittel, K., \& Tozzi, J. E. (2020). Bilateral acetabular fractures treated with delayed total hip arthroplasty. Arthroplast Today., 6(2), 236-240.

15. Ingle, M., Bhalotia, A., \& Chandele, V. (2018). Bilateral anterior column acetabulum fracture following road traffic accident: A rare presentation. Journal of Orthopaedic Case Reports., 8(5), 72-74.

16. Khalily, C., Ilizaliturri, V. M., Jr., \& Lester, D. K. (2002). Bilateral pathologic fractures of the hip and acetabulum treated with cementless total hip arthroplasty. The Journal of Arthroplasty., 17(5), 664-666.

17. Langenhan, R., Hohendorff, B., Trobisch, P., \& Probst, A. (2014). Simultaneous bilateral humeral head disclocation and acetabular fracture. A rare manifestation after hypoglycemia-induced seizures. Der Unfallchirurg, 117(8), 747-751.

18. Magu, N. K., Gogna, P., Singh, A., Singla, R., Rohilla, R., Batra, A., et al. (2014). Long term results after surgical management of posterior wall acetabular fractures. Journal of Orthopaedics and Traumatology, 15(3), 173-179.

19. McEwan, L. M. (2003). Unsuspected bilateral central acetabular fractures diagnosed with nuclear scintigraphy. Australasian Radiology, 47(4), 447-449.

20. McMahon, S. E., \& Cusick, L. A. (2017). Total hip replacement in complex acetabular fractures using a coned hemipelvic acetabular component. European Journal of Orthopaedic Surgery \&amp; Traumatology, 27(5), 631-636.

21. Medici, A., Di Salvatore, M. G., Pezzella, R., Fidanza, A., De Simone, A. M., \& Calvisi, V. (2020). Bilateral fractures of acetabulum in a young girl with osteogenesis imperfecta and epilepsy. Trauma Case Reports, 25, 100265.

22. Meena, U. K., Meena, D. S., Behera, P., \& Meel, O. S. (2014). Bilateral central acetabular fracture dislocation in a young patient due to seizure activity: A case report and review of the literature. Chinese Journal of Traumatology, 17(6), 364-366.

23. Mohamed, N., Makaranka, S., Cheema, K., \& Harnett, P. (2019). Bilateral acetabular fractures induced by an epileptic seizure in a paediatric patient: A unique case and its management. BMJ Case Reports, 12(8), e230143.

24. Nehme, A. H., Matta, J. F., Boughannam, A. G., Jabbour, F. C., Imad, J., \& Moucharafieh, R. (2012). Literature review and clinical presentation of bilateral acetabular fractures secondary to seizure attacks. Case Reports in Orthopedics., 2012, 240838.

25. Nodzo, S. R., Hohman, D. W., \& Galpin, R. D. (2012). Bilateral acetabular fractures in an adolescent after low-energy trauma. Pediatric Emergency Care, 28(6), 568-569.

26. Olcay, E., Adanır, O., Ozden, E., \& Barış, A. (2012). Bilateral asymmetric traumatic hip dislocation with bilateral acetabular fracture: Case report. Ulusal Travma ve Acil Cerrahi Dergisi., 18(4), 355-357.

27. Rommens, P. M., Bossche, M. V., Fevery, S., \& Blum, J. (1996). Bilateral acetabulum fractures of the anterior column and vertebral body fracture after alcohol-induced seizure. Der Unfallchirurg, 99(9), 704-707.

28. Rosa, M. A., Maccauro, G., \& D’Arienzo, M. (1999). Bilateral acetabular fracture without trauma. International Orthopaedics, $23(2), 120-121$.

29. Sahin, O., Ozturk, C., Dereboy, F., \& Karaeminogullari, O. (2007). Asymmetrical bilateral traumatic hip dislocation in an adult with bilateral acetabular fracture. Archives of Orthopaedic and Trauma Surgery, 127(8), 643-646.

30. Schachter, A. K., Roberts, C. S., \& Seligson, D. (2003). Occult bilateral acetabular fractures associated with high-energy 
trauma and osteoporosis. Journal of Orthopaedic Trauma, 17(5), 386-389.

31. Shenouda, R., Carter, S., Wang, K., Chow, Y., Topliss, D. J., \& Esser, M. P. (2016). Multi-trauma secondary to hypocalcaemiainduced seizure: A case report. Trauma Case Reports., 6, 8-12.

32. Sheth, H., Salunke, A. A., Panchal, R., Chokshi, J., Nambi, G. I., Singh, S., et al. (2016). Simultaneous bilateral shoulder and bilateral central acetabular fracture dislocation: What to do? Chinese Journal of Traumatology, 19(1), 59-62.

33. Singh, A. P., Sidhu, A. S., \& Singh, A. P. (2010). Traumatic bilateral hip dislocation with bilateral sciatic nerve palsy. Chinese Journal of Traumatology, 13(2), 126-128.

34. Stevens, J. M., Shiels, S., Whitehouse, M. R., Ward, A. J., Chesser, T. J., \& Acharya, M. (2020). Bilateral acetabular fractures: Mechanism, fracture patterns and associated injuries. Journal of Orthopaedics, 18, 28-31.

35. Takahashi, Y., Ohnishi, H., Oda, K., \& Nakamura, T. (2007). Bilateral acetabular fractures secondary to a seizure attack caused by antibiotic medicine. Journal of Orthopaedic Science, 12(3), 308-310.

36. Taylor, B. C., \& Poka, A. (2012). Definitive treatment of bilateral acetabular and pelvic ring injuries using external fixation. The Iowa Orthopaedic Journal., 32, 220-223.

37. Tempelaere, C., Diviné, P., \& Bégué, T. (2019). Early simultaneous bilateral total hip arthroplasty for the management of bilateral acetabular fracture in an elderly patient. Arthroplast Today., 5(2), 139-144.

38. Trost, P., Kollersbeck, C., Pelitz, M., Walcher, T., \& Genelin, F. (2013). Bilateral acetabulum fracture after suffering sport trauma. Der Unfallchirurg, 116(7), 653-657.

39. Vaishya, R., Kumar, R., \& Maharjan, R. R. (2014). Percutaneous fixation of bilateral anterior column acetabular fractures: A case report. Apollo Medicine., 11(1), 52-55.

40. Van Heest, A., Vorlicky, L., \& Thompson, R. C., Jr. (1996). Bilateral central acetabular fracture dislocations secondary to sustained myoclonus. Clinical Orthopaedics and Related Research, 324, 210-213.

41. Yngstrom, K., Stapleton, E., Aberman, Z., \& Galos, D. (2020). Bilateral acetabular fractures associated with seizures: A report of 2 cases. JBJS Case Connect., 10(1), e0190.

42. Citak, M., Backhaus, M., Kälicke, T., Brosch, B., Horch, C., Muhr, G., et al. (2010). Querschnittslähmung nach isolierter Rückenmarksruptur-eine seltene Verletzung. Zeitschrift für Orthopädie und Unfallchirurgie, 148(06), 662-665.

43. Williams, T. R., Puckett, M. L., Denison, G., Shin, A. Y., \& Gorman, J. D. (2002). Acetabular stress fractures in military endurance athletes and recruits: Incidence and MRI and scintigraphic findings. Skeletal Radiology, 31(5), 277-281.

44. Judet, R., Judet, J., \& Letournel, E. (1964). Fractures of the acetabulum: Classification and surgical approaches for open reduction. Preliminary report. The Journal of Bone and Joint Surgery. American Volume., 46, 1615-1646.

45. Giordano, V., Acharya, M. R., Pires, R. E., \& Giannoudis, P. V. (2020). Associated both-column acetabular fracture: An overview of operative steps and surgical technique. Journal of Clinical Orthopaedics and Trauma., 11(6), 1031-1038.

46. Kelly, J., Ladurner, A., \& Rickman, M. (2020). Surgical management of acetabular fractures - A contemporary literature review. Injury, 51(10), 2267-2277.
47. Kumar, A., Shah, N., Kershaw, S., \& Clayson, A. (2005). Operative management of acetabular fractures: A review of 73 fractures. Injury, 36(5), 605-612.

48. Butler, B. A., Lawton, C. D., Hashmi, S. Z., \& Stover, M. D. (2019). The relevance of the judet and letournel acetabular fracture classification system in the modern era: a review. Journal of Orthopaedic Trauma, 33(Suppl 2), S3-s7.

49. Cimerman, M., Kristan, A., Jug, M., \& Tomaževič, M. (2020). Fractures of the acetabulum: from yesterday to tomorrow. International Orthopaedics., 45, 1057-1064.

50. Alton, T. B., \& Gee, A. O. (2014). Classifications in brief: Letournel classification for acetabular fractures. Clinical Orthopaedics and Related Research, 472(1), 35-38.

51. de Bakker, H. M., Tijsterman, M., Kubat, B., Soerdjbalie-Maikoe, V., van Rijn, R. R., \& de Bakker, B. S. (2018). Postmortem radiological case series of acetabular fractures after fatal aviation accidents. Forensic Science, Medicine and Pathology, 14(1), 62-69.

52. Giannoudis, P. V., Grotz, M. R., Papakostidis, C., \& Dinopoulos, H. (2005). Operative treatment of displaced fractures of the acetabulum. A meta-analysis. The Journal of Bone and Joint Surgery. British Volume., 87(1), 2-9.

53. Ferguson, T. A., Patel, R., Bhandari, M., \& Matta, J. M. (2010). Fractures of the acetabulum in patients aged 60 years and older: An epidemiological and radiological study. Journal of Bone and Joint Surgery. British Volume, 92(2), 250-257.

54. Papadakos, N., Pearce, R., \& Bircher, M. D. (2014). Low energy fractures of the acetabulum. Annals of the Royal College of Surgeons of England, 96(4), 297-301.

55. Pearson, J. R., \& Hargadon, E. J. (1962). Fractures of the pelvis involving the floor of the acetabulum. Journal of Bone and Joint Surgery. British Volume., 44B, 550-561.

56. Iwamoto, H., Nakamura, K., Watanabe, D., Yanaka, K., Ainoya, T., \& Yamamoto, T. (2018). Nontraumatic acetabular fracture sustained during convulsive seizure following surgery for an unruptured intracranial aneurysm: A case report. NMC Case Report Journal., 5(2), 57-60.

57. Zhao, D., Cheng, P., \& Zhu, B. (2016). Epilepsy and fracture risk: A meta-analysis. International Journal of Clinical and Experimental Medicine., 9, 564-569.

58. Mears, D. C. (2008). Total hip arthroplasty for acute acetabular fractures. Seminars in Arthroplasty: JSES., 19(4), 291-302.

59. Shah, N., Gill, I. P., Hosahalli Kempanna, V. K., \& Iqbal, M. R. (2020). Management of acetabular fractures in elderly patients. Journal of Clinical Orthopaedics and Trauma., 11(6), 1061-1071.

60. Hull, J. B., Raza, S. A., Stockley, I., \& Elson, R. A. (1997). Surgical management of fractures of the acetabulum: The Sheffield experience 1976-1994. Injury, 28(1), 35-40.

61. Kilinc, C. Y., Acan, A. E., Gultac, E., Kilinc, R. M., Hapa, O., \& Aydogan, N. H. (2019). Treatment results for acetabulum fractures using the modified Stoppa approach. Acta Orthopaedica et Traumatologica Turcica, 53(1), 6-14.

Publisher's Note Springer Nature remains neutral with regard to jurisdictional claims in published maps and institutional affiliations. 